\title{
Actividad física, estado nutricional y consumo habitual de alimentos en
}

escolares del norte de Chile

DOI: 10.17533/udea.penh.v21n1a03

PERSPECTIVAS EN NUTRICIÓN HUMANA

ISSN 0124-4108

Escuela de Nutrición y Dietética, Universidad de Antioquia. Medellín, Colombia

Vol. 21, N. ${ }^{\circ}$, enero-junio de 2019, pp. 27-40.
Artículo recibido: 22 de marzo de 2019 Aprobado: 6 de agosto de 2019

\section{Guido Clemente Solari Montenegro"*; Monserrat Elliet Rivera Iratchet²; Bruno Antonio Solari Vega $^{3}$; Ana María Wall Pacheco ${ }^{4}$; María Belén Peralta Peña}

\section{- -Resumen}

Antecedentes: Chile triplicó la prevalencia de obesidad en estudiantes de primero básico de 5,8 \% (1987) a 17 \% (2000). Objetivo: identificar hábitos de actividad física, estado nutricional y consumo de alimentos en escolares de primer año básico de la ciudad de Taltal, Chile. Materiales y métodos: estudio observacional, descriptivo, transversal, en 94 estudiantes de 6,5 $\pm 0,5$ años de Taltal, Chile, en quienes se exploró actividad física, consumo de alimentos y estado nutricional según IMC y perímetro de cintura. Resultados: según IMC, el 58,5\% del total estudiado tenía algún grado de exceso, que incluía $23,4 \%$ de obesidad y $5,3 \%$ de obesidad severa, más prevalentes en hombres ( $p=0,035$ y $p=0,014$ respectivamente) y $28 \%$ de sobrepeso sin diferencia por sexo ( $p>0,05)$. En las combinaciones de almuerzo y comida, ingieren $78 \%$ de alimentos altos en grasa y $16,8 \%$ bajos en grasa. Solo el $41,5 \%$ de los sujetos realizaban actividades físicas sistemáticas, sin diferencias según sexo $(p<0,05)$. Conclusión: según IMC, el $58,3 \%$ tiene sobrepeso u obesidad, esta última más prevalente entre hombres. Ambos sexos consumen preferentemente alimentos altos en grasas y el $66 \%$ realiza actividad física compatible con sedentarismo.

Palabras clave: conducta alimentaria, ejercicio, nutrición, sobrepeso, obesidad.

$1^{*} \quad$ Autor de correspondencia. Doctor en Investigación en Actividad Física. Académico, Klgo. Facultad de Cs. de la Salud, Universidad de Antofagasta, Chile. Avda. Angamos 601, cel. 97733835. guido.solari@uantof.cl

2 Magíster en Neurorrehabilitación. Académico, Klgo. Facultad de Cs. de la Salud, Universidad de Antofagasta, Chile.

3 Médico. Docente de Internado, Facultad de Medicina y Odontología, Universidad de Antofagasta, Chile.

4 Magíster en Salud Pública. Académico, nutricionista. Facultad de Cs. de la Salud, Universidad de Antofagasta, Chile.

5 Magíster en Nutrición y Alimentación. Académico, nutricionista. Facultad de Cs. de la Salud, Universidad de Antofagasta, Chile.

Cómo citar este artículo: Solari-Montenegro GC, Rivera-Iratchet ME, Solari-Vega BA, Wall-Pacheco AM, Peralta-Peña MB. Actividad física, estado nutricional y consumo habitual de alimentos en escolares del norte de Chile. Perspect Nutr Humana. 2019;21:27-40. DOI: 10.17533/udea.penh.v21n1a03 


\section{Physical Activity, Nutritional Status, and Habitual Dietary Intake in School Children in the North of Chile}

\section{Abstract}

Background: The prevalence of obesity in school-aged children in Chile tripled from $5.8 \%$ in 1987 to $17 \%$ in 2000. Objective: Identify physical activity habits, nutritional status, and food consumption patterns in first graders in the city of Taltal, Chile. Materials and Methods: Observational, descriptive, cross-sectional study of 94 students $6.5 \pm 0.5$ years of age in Taltal, Chile to explore physical activity, food intake, and nutritional status as measured by BMI and waist circumference. Results: According to BMI, $58.5 \%$ of the sample had excess weight; $23.4 \%$ obese and $5.3 \%$ severely obese, with higher prevalences observed in males ( $p=0.035$ and $p=0.014$ respectively). $28 \%$ of both males and females were overweight ( $p>0.05$ ). Of the foods consumed at snack and lunch, $78 \%$ were high in fat and $16.8 \%$ low in fat content. Only $41.5 \%$ of subjects both male and female $(p<0.05)$ participated in regular physical activity. Conclusions: As measured by BMI, $58.3 \%$ of students were overweight or obese, with obesity more prevalent in males. Both sexes consumed a majority of foods with a high fat content and $66 \%$ of students are sedentary.

Keywords: Dietary habits, physical activity, nutrition, overweight, obesity.

\section{INTRODUCCIÓN}

A partir de la década del ochenta del siglo pasado, la obesidad en estudiantes de primero básico se triplicó, en doce años evolucionó de 5,8 \% en 1987, a $17 \%$ en 2000. En los preescolares, la prevalencia se duplicó entre $2004(5,7 \%)$ y 2006 $(11,6 \%)$; siendo las regiones del sur aquellas con más alta prevalencia de obesidad regional elevándose, algunas, por sobre el $30 \%$ (1-3).

Algunos autores han planteado que, en el país, el incremento en la ingesta de alimentos hipercalóricos y la jornada escolar completa han contribuido al aumento de la prevalencia del sobrepeso, considerando que el chileno a partir de los años ochenta del siglo pasado modificó su dieta hacia una de mayor consumo de grasas (13,9 kg/pers/ año en 1975 a 16,7 kg en 1995), alimentos elaborados (30,2 kg/pers/año en 1975 a 39,2 kg en 1995) y menor consumo de pescados, legumbres y verduras (4-6). Este fenómeno está altamente relacionado con los cambios en el estilo de vida y la alimentación de los escolares.

Por otra parte, en 2015 el Ministerio de Educación Pública de Chile, mediante la Agencia de Calidad de la Educación, reportó que había diferencia por sexo en la actividad física y que las mujeres de todos los grupos socioeconómicos tenían más baja actividad que los hombres; señaló, además, que las escuelas constituían una oportunidad para mejorar la condición física de los estudiantes, compensar el sedentarismo derivado de la actividad física de bajo costo, formar hábitos saludables de vida y mejorar los años de vida saludables (7).

La información en materia de estado nutricional, consumo de alimentos y hábitos de actividad física en escolares de la segunda región de Chile es una cuestión pendiente. Los datos publicados en artículos científicos y documentos gubernamentales oficiales se han referido principalmente a la ciudad de Santiago de Chile y, eventualmente, a algunas otras regiones distintas de la segunda región. 
La hipótesis de trabajo del presente artículo propone que la alta prevalencia de obesidad y sobrepeso de los escolares de primer año básico de la ciudad de Taltal, Chile, se vincula con la calidad y cantidad de alimentos ingeridos, y con la escasa práctica de actividad física en el colegio y fuera de él. Por ello, el estudio tuvo como objetivo identificar los hábitos de actividad física, el estado nutricional y el consumo de alimentos de los escolares de primer año básico.

\section{MATERIALES Y MÉTODOS}

Utilizando un diseño observacional, descriptivo y transversal se exploró los hábitos de actividad física, consumo de alimentos, estado nutricional y algunos indicadores antropométricos del universo total de estudiantes de nivel básico de la ciudad de Taltal ( $n=94,50$ hombres y 44 mujeres), siendo la edad promedio de ellos de 6,4 años (Tabla 1). Todos los estudiantes eran alumnos regulares de las tres escuelas públicas existentes en la ciudad.

Tabla 1. Edad y características antropométricas según sexo

\begin{tabular}{|c|c|c|c|c|}
\hline & $\begin{array}{l}\text { Mujeres } \\
\qquad n=44\end{array}$ & $\begin{array}{l}\text { Hombres } \\
\qquad n=50\end{array}$ & Total & Valores de $\mathrm{p}^{*}$ \\
\hline$n(\%)$ & $44(47 \%)$ & $50(53 \%)$ & 94 & \\
\hline Edad (años) $\mathrm{X} \pm \mathrm{DE}$ & $6,5 \pm 0,5$ & $6,5 \pm 0,5$ & 94 & 1 \\
\hline \multicolumn{5}{|l|}{ Antropometría } \\
\hline Talla (cm) $X_{ \pm} \mathrm{DE}$ & $121,4 \pm 5,6$ & $120,7 \pm 4,8$ & 94 & $0,518^{*}$ \\
\hline $\mathrm{P}=$ Perímetro de cintura $(\mathrm{cm})$ & $58,6 \pm 5,2$ & $59,0 \pm 6,2$ & 94 & $0,737^{\star}$ \\
\hline Peso corporal $(\mathrm{kg}) \mathrm{X} \pm \mathrm{DE}$ & $26,2 \pm 4,2$ & $26,3 \pm 4,9$ & 94 & $0,916^{*}$ \\
\hline IMC (kg/talla2) $\mathrm{X} \pm \mathrm{DE}$ & $17,7 \pm 2,4$ & $18,0 \pm 2,4$ & 94 & $0,546^{*}$ \\
\hline \multicolumn{5}{|l|}{ Estado nutricional } \\
\hline Obesidad severa n (\%) & $1(2,3)$ & $4(18)$ & $5(5,3)$ & $0,014^{\dagger}$ \\
\hline Obesidad n (\%) & $6(13,6)$ & $16(32)$ & $22(23,4)$ & $0,035^{\dagger}$ \\
\hline Sobrepeso n (\%) & $15(32,1)$ & $13(26)$ & $28(29,8)$ & $0,515^{\dagger}$ \\
\hline Eutrofia n (\%) & $21(47,7)$ & $17(34)$ & $38(40,4)$ & $0,177^{\dagger}$ \\
\hline Bajo peso n (\%) & $1(2,3)$ & $0,0(0,0)$ & $1(1,1)$ & $0,280^{\dagger}$ \\
\hline Desnutrición n (\%) & $0,0(0,0)$ & $0,0(0,0)$ & $0(0,0)$ & - \\
\hline \multicolumn{5}{|l|}{ Clasificación de OA } \\
\hline Obesidad abdominal (\%) & $4(4,3)$ & $7(7,4)$ & $11(11,7)$ & $0,003^{\ddagger}$ \\
\hline Riesgo de OA & $12(12,8)$ & $18(19,1)$ & $30(31,9)$ & $0,418^{\ddagger}$ \\
\hline Normal & $27(28,7)$ & $23(24,5)$ & $50(53,2)$ & $0,654^{\ddagger}$ \\
\hline
\end{tabular}

$\mathrm{OA}=$ obesidad abdominal.

* Valor de p de la comparación de la distribución de valores antropométricos por sexo mediante diferencia de dos medias de muestras provenientes de distribuciones normales.

† Valor de p de la comparación de la distribución del estado nutricional según IMC, por sexo mediante prueba de hipótesis de diferencia de proporciones binomiales.

₹ Valor de p de la comparación de la clasificación de obesidad abdominal por sexo mediante prueba de hipótesis de diferencia de proporciones binomiales. 


\section{Evaluación antropométrica del estado nutricional}

El peso y la estatura de los escolares fueron medidos por nutricionistas capacitadas, que utilizaron una báscula de columna marca $\mathrm{Seca}^{\circledR} 220$, un tallímetro portátil marca Seca ${ }^{\circledR} 213$ y una cinta métrica metálica marca Cescorf ${ }^{\circledR}$ para medir el perímetro abdominal o perímetro de cintura $(\mathrm{PC})$, según la norma para la evaluación nutricional de niños, niñas y adolescentes de 5-19 años de edad, publicada por el Ministerio de Salud en el año 2016. Para la evaluación de la obesidad abdominal, se utilizó el PC y se usaron como valores de referencia los establecidos según sexo y edad por el Ministerio de Salud de Chile (2016). La clasificación se hizo de acuerdo con los puntos de corte expresados en percentiles, definidos por el mismo organismo, asi: obesidad abdominal mayor a p 90 (>p 90); cuando el perímetro de cintura se ubica entre el $p 75$ y p 90, se consideró riesgo de obesidad abdominal y cuando el valor estaba por debajo del p 75 se consideró normal (8).

Se calculó el Î́ndice de Masa Corporal (IMC). Para la clasificación del estado nutricional según el IMC, se comparó el valor de cada escolar con los patrones de referencia de uso internacional, y se utilizaron los puntos de corte propuestos por el Ministerio de Salud de Chile, publicados en las normas para la evaluación nutricional de niños y niñas y adolescentes de 5 a 19 años de edad, que establecen seis categorías según la desviación estándar en la que se ubiquen los valores de IMC, así: obesidad severa: $>+3$; obesidad: $>+2 \mathrm{a}+2,9$; sobrepeso: $>1,0$ a 1,9; eutrofia: $+0,9$ a $-0,9$; bajo peso: $<-1$ a $-1,9$; desnutrición: $<-2$ (8-9).

\section{Evaluación de hábitos de consumo de alimentos}

Para estimar los hábitos de alimentos, nutricionistas capacitadas aplicaron a cada escolar una encuesta de hábitos de ingesta previamente utilizada en una publicación sobre la misma materia, la cual exploró el número de comidas diarias y las características cualitativas de los desayunosonces, almuerzos-cena, refrigerios (alimentos que se llevan al colegio y que se ingieren entre las horas de clases) y alimentos extras (alimentos ingeridos fuera del horario de comidas habituales) (10). Estas comidas fueron clasificadas como altas en grasa, moderadas en grasa y bajas en grasa (ver Anexo 1).

Para obtener información confiable sobre la cantidad de alimentos consumidos por los escolares, las nutricionistas utilizaron un libro con imágenes de distintas porciones de alimentos, con transformación a gramos validada en escolares chilenos. En el caso de los alimentos envasados, las nutricionistas consultaron por la marca del producto para obtener la cantidad directamente del envase mediante.

\section{Evaluación de la actividad física}

Para estimar los hábitos de actividad física, se consultó a los escolares mediante una encuesta utilizada en una publicación similar a la actual (10), en la cual se evaluó el tipo de actividad, las actividades de gasto mínimo, el total de cuadras caminadas, el tiempo dedicado a los juegos recreativos y las actividades físicas sistemáticas, asignando un puntaje de acuerdo con la cuantificación de cada una de las actividades (Anexo 2).

Las definiciones de sedentarismo son variadas, algunas investigaciones sugieren que cumplen con esta característica aquellos individuos que practican actividad física sistemática con una frecuencia menor a tres veces por semana, con una duración menor a veinte minutos por cada una de esas sesiones (11-12).

\section{Análisis estadístico}

Mediante descripción estadística y estimación de media, desviación estándar, varianza y valores 
mínimo y máximo, se exploró la distribución de los datos de edad, talla, PC, peso corporal e IMC, analizándolos también mediante el cálculo de diferencia de medias. Para la comparación según sexo del estado nutricional, combinaciones de alimentos, consumo de alimentos extra, se aplicó la prueba de hipótesis de diferencias de proporciones binomiales, usando el estadístico Z y un nivel de confianza del $95 \%$.

\section{Consideraciones éticas}

El presente estudio fue sometido a la evaluación del Comité de Ética acreditado de la Universidad de Antofagasta. A los padres, apoderados o tutores legales se les solicitó - por exigencia de dicho Comité- la aprobación previa del consentimiento escrito en el cual se informaba el objetivo del estudio y la descripción de los exámenes que se harían a su hijo, como también la participación para responder las encuestas. A los menores se les solicitó el asentimiento respecto de los procedimientos de evaluación de que serían objeto, explicándoles verbalmente los procedimientos, una vez aceptados el consentimiento y el asentimiento se procedió a la inclusión de cada menor como parte del estudio.

\section{RESULTADOS}

\section{Evaluación antropométrica}

La distribución de los escolares según sexo, edad, talla, PC, peso corporal e IMC de hombres y mujeres fue similar en materia de valores promedio, se observó valores levemente superiores en los hombres en cuanto a PC, IMC y peso (Tabla 1); no obstante, los valores de $\mathrm{p}$ obtenidos mediante la diferencia de medias no mostraron diferencias estadísticamente significativas.

En la población total de estudiantes de primer año básico evaluada, 58,3 \% presentó algún grado de exceso de peso con un porcentaje de obesi- dad importante (23,4\%), incluso obesidad severa (5,3\%). Según sexo, en los hombres predominó la obesidad y la obesidad severa en tanto que en las mujeres existió una mayor prevalencia de sobrepeso. El IMC fue normal en la mitad de las mujeres y solo en un tercio de los hombres. Acorde a la distribución del estado nutricional según IMC por sexo, solo se hallaron diferencias significativas para las clasificaciones de obesidad severa $(p=0,014)$ y obesidad ( $p=0,035)$ y fueron los hombres quienes presentaron los valores más altos (Tabla 1).

El $50 \%$ de la población explorada clasificó como normal según $\mathrm{PC}$, un $30 \%$ clasificó con riesgo de obesidad abdominal y un $11 \%$ con obesidad abdominal. Según los valores de $p$, no se observó diferencias estadísticamente significativas entre sexos para ninguna de las clasificaciones (Tabla 1).

\section{Hábitos de consumo de alimentos}

Al analizar las encuestas de hábitos de alimentación se observó que —según el número de comidas diarias- la mitad de los escolares (52,6\%) ingieren tres comidas al día, un 44,2\% consume cuatro o más y solo el 2,1\% dos comidas diarias. Las mujeres fueron quienes refirieron ingerir regularmente tres comidas al día y los hombres cuatro. Al agruparlos según las combinaciones de comidas, se observó en ambos sexos las combinaciones de desayuno-onces y almuerzo-comida altos en grasas (leche entera, pan amasado, hallulla, ${ }^{1}$ pan con queso, mantequilla o mortadela, te o café puro, hotdog, pizza, productos de pastelería, huevo frito, manjar, paté y arrollado) y moderados en grasas (carnes, arroz, fideos, puré, pan, papas, sopas, compotas de fruta, jaleas, yogurt, jugo en polvo y bebidas dietéticas) respectivamente. No obstante, fueron los hombres quienes prefirieron mantener el mismo patrón de ingesta, pero con porcentajes más elevados en las combinaciones antes descritas.

\footnotetext{
1 Un tipo de pan blanco, elaborado a base de harina de trigo.
} 
Al comparar la distribución de las diversas combinaciones de alimentos según sexo, y obtener el valor de $p$, se halló — con un nivel de confianza del $95 \%$ - que no existen diferencias estadísticamente significativas entre hombres y mujeres para la combinación desayuno-onces altos en grasa $(p=0,663)$, como tampoco para los moderados en grasa $(p=0,719)$, pero sí en los bajos en grasa $(p=0,000)$, valor que fue mayor en las mujeres (Figura 1).

Al organizar a los estudiantes según el tipo de refrigerio consumido en la escuela, se observó que prácticamente el $80 \%$ de ellos consumía preferentemente alimentos altos en grasa (papas fritas, pasteles, chocolates, confites, snacks, helados, alfajores, negritas, bebidas gaseosas y masas fritas -conocidas en Chile como sopaipillas-) y moderados en grasa (sándwich de palta o pollo, galletas, nueces, cereales, jugos azucarados). Respecto de los alimentos extras, la misma proporción de estudiantes (80\%) consumía alimentos preferentemente altos en grasas (dulces, chocolates, papas fritas y similares, bebidas gaseosas, productos de pastelería, snacks, helados, masas fritas, rosquillas —conocidas en Chile como picarones-, pasteles, sándwich jamón o queso) y moderados en grasas (galletas, sándwich de ave, atún, mermelada y pan solo). En promedio, solo el $10 \%$ de los estudiantes consumió refrigerios y alimentos extras bajos en grasas (fruta, jugos de fruta yogurt, agua).

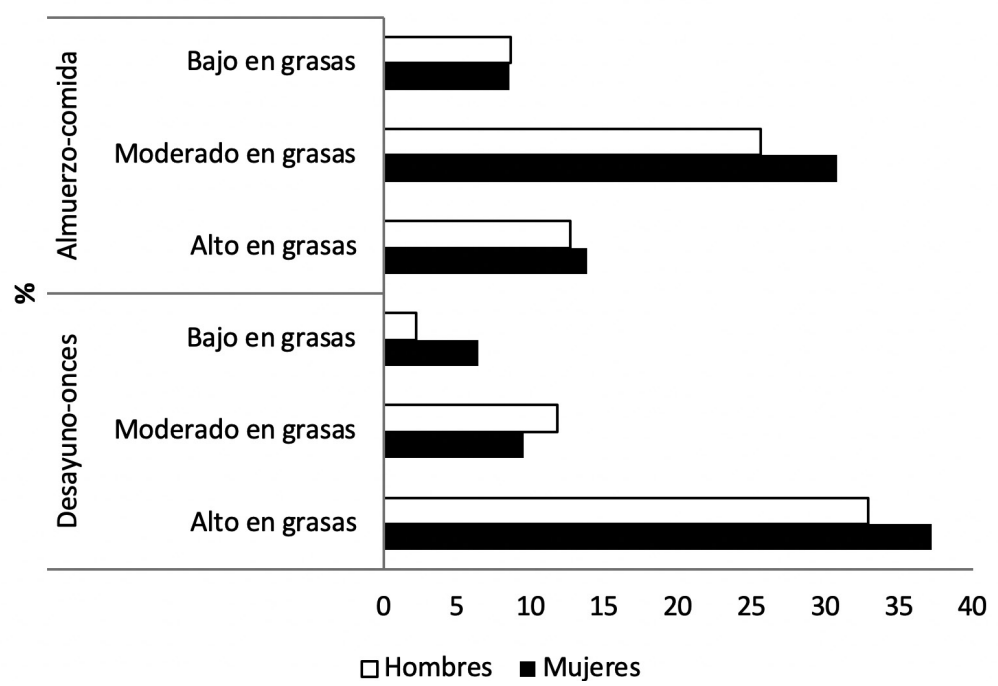

Figura 1. Distribución de los estudiantes según combinaciones de alimentos por tipo de comida y sexo.

Al comparar la distribución de las diversas combinaciones de refrigerios y alimentos extras según sexo y obtener el valor de $p$-con un nivel de confianza del $95 \%$ - no se encontraron diferencias en refrigerios altos en grasa ( $p=0,891)$, ni en moderados en grasa $(p=0,133)$ ni en los bajos en grasa $(p=0,392)$. Tampoco se encontraron diferencias por sexo — según la misma prueba - en alimentos extra altos en grasa $(p=0,638)$ ni entre los moderados en grasa $(p=0,649)$, pero sí entre los bajos en grasa $(p=0,000)$, con mayor proporción entre las mujeres (Figura 2).

\section{Evaluación de la actividad física}

Al analizar el número de cuadras caminadas diariamente se encontró que $50 \%$ de los encuestados caminaba menos de cinco, y únicamente el $1 \%$ lo hacía en más de 15 cuadras. Independiente del sexo, la encuesta incorporó consultas en relación con el gasto mínimo de energía (Tabla 2). 


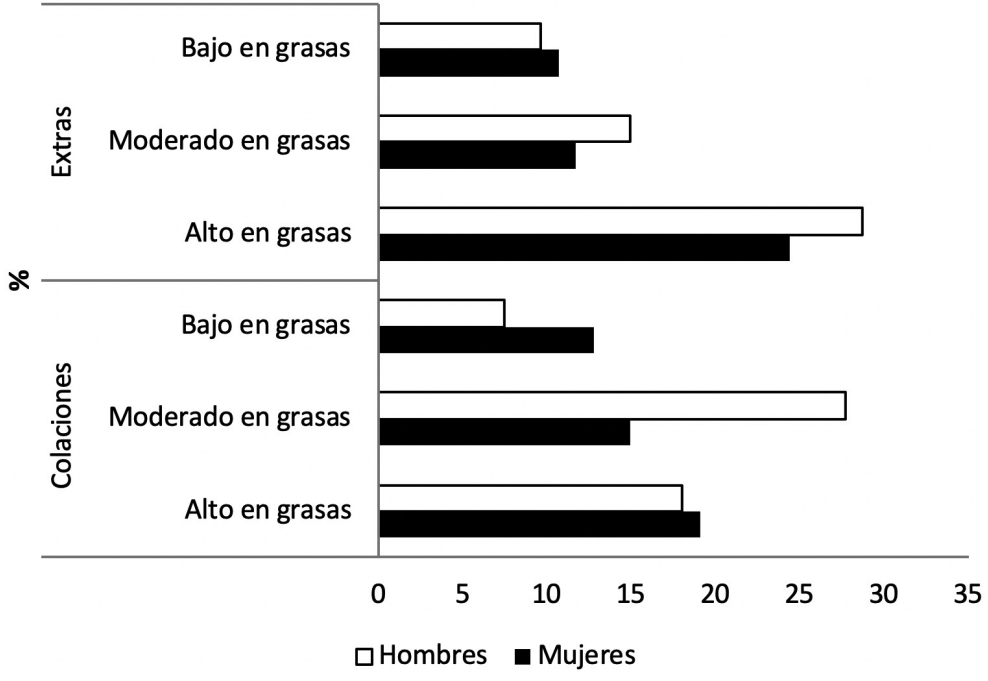

Figura 2. Distribución de los estudiantes según combinaciones de alimentos en refrigerios y alimentos extra.

Tabla 2. Distribución de los estudiantes según cuadras caminadas diarias, juegos recreativos diarios y actividad física sistemática semanal según sexo

\begin{tabular}{lcccc}
\hline \multicolumn{1}{c}{ Variable } & Hombres & Mujeres & Total & Valor de $\mathrm{p}^{*}$ \\
\hline$\geq 5$ cuadras caminadas/día, $\mathrm{n}(\%)$ & $\mathrm{n}=\mathbf{4 4}$ & $\mathrm{n}=\mathbf{5 0}$ & $\mathrm{n}=\mathbf{9 4}$ & 1 \\
$\geq 30$ minutos de juegos recreativos/día, $\mathrm{n}(\%)$ & $10(23,4)$ & $12(23,4)$ & $22(23,4)$ & 0,748 \\
$\geq 2$ horas de actividad física sistémica/semana, $\mathrm{n}(\%)$ & $17(38,3)$ & $18(35,1)$ & $35(37,2)$ & 0,776 \\
\hline
\end{tabular}

* Valor de p de la comparación de la distribución de cuadras caminadas diariamente, juegos recreativos diarios y actividad física sistemática semanal según sexo, mediante prueba de hipótesis de diferencias de proporciones binomiales.

Respecto de otras actividades desarrolladas por los estudiantes, se observó que independiente del sexo todos tenían conductas muy similares. Ellos distribuían proporcionalmente su tiempo (menos de seis horas diarias) para las tareas de gasto mínimo, tales como ver televisión, realización de tareas escolares y dedicación al celular y al computador.

Finalmente, al comparar la actividad física explorada (cuadras caminadas, juegos recreativos diarios, actividad física sistemática semanal) según sexo, considerando los valores de $p$, se puede afirmar - con un nivel de confianza del $95 \%$ - que no existen diferencias estadísticamente significativas entre ambos grupos (Tabla 2).

\section{DISCUSIÓN}

La prevalencia de obesidad en los escolares evaluados es alta (27\%), con valores mayores en los hombres. En cuanto al consumo, la única diferencia estadística según sexo se encontró en el consumo de alimentos extra bajos en grasa, que es mayor en las mujeres. La actividad física es baja tanto en hombres como en mujeres: menos de la mitad de los sujetos estudiados camina cinco cuadras o más diariamente; menos del $40 \%$ dedica 30 minutos o más por día a los juegos recreativos, y solo el $41,5 \%$ realiza dos horas o más de actividad física sistemática semanal. 
La prevalencia de obesidad $(28,3 \%)$ es similar a la encontrada en la población de primer año básico por otros autores chilenos (13), quienes evaluaron escolares de la ciudad de La Serena y hallaron una prevalencia del $25,5 \%$, de modo similar a lo reportado a nivel nacional por la Junta Nacional de Auxilio Escolar y Becas (25,3\%) en el mismo grupo de escolares de primer año básico (14).

Un 29,5\% de la población reportada en este artículo es clasificada con sobrepeso, cifra similar al 24,6 \% hallado por Lancellotti et al. (21) en 2016. En el presente estudio, predominó —en los hombres - la obesidad y la obesidad severa, pero en las mujeres la mayor prevalencia correspondió al sobrepeso, similar a lo que reportaron otros autores chilenos (13-15).

En el grupo explorado se encontró una prevalencia de sobrepeso de $24,6 \%$, cifra que difiere con lo reportado por Ulloa et al. (15) en 2010, aunque concuerda en la prevalencia del sobrepeso mayor en mujeres (22\%). La situación epidemiológica actual releva la importancia del exceso de peso y la importancia del control periódico de bajo peso y talla baja.

En materia de PC, un $32 \%$ de las mujeres y solo un $22 \%$ de los hombres califican como normales; estos datos coinciden con los del estudio de Benjumea et al. (16), quienes al explorar la circunferencia de la cintura en niños escolares manizaleños encontraron valores de PC menores en las niñas que en los niños. La magnitud de la información reportada en el presente artículo difiere de la hallada por otros autores chilenos, quienes encontraron hasta un $76,5 \%$ de obesidad abdominal en una muestra de niños y adolescentes chilenos, aunque la adecuación ponderal se evaluó por el puntaje Z del IMC del referente CDC-NCHS (17).
Es necesario relevar lo anterior, considerando la evidencia epidemiológica disponible, la cual vincula la adiposidad abdominal con el incremento del riesgo de síndrome metabólico en la niñez y adolescencia y eventualmente de enfermedad cardiovascular, dislipidemia y diabetes mellitus 2 (18). Además, la coincidencia de estudios respecto a que en niños el PC es mejor predictor de riesgo cardiovascular y metabólico que el IMC, Io cual apoya la conveniencia de incluir el PC en la evaluación del niño como tamizaje del riesgo metabólico derivado de la grasa abdominal (19).

Si se considera el infarto agudo al miocardio y la insuficiencia renal crónica como algunas de las principales complicaciones de las enfermedades antes mencionadas, especial relevancia asumen estas proyecciones en la ciudad del estudio, donde se cuenta con un hospital comunitario y un centro de diálisis modular, lo que eleva los costos de inversión y tratamiento para poder tener la cobertura necesaria a potenciales usuarios.

Respecto a las combinaciones de alimentos tanto para desayuno-onces como almuerzos-comidas, se encuentra que los escolares del presente estudio presentan una conducta similar a la hallada por Zarceño (20), quien concluyó en su estudio que los alimentos más consumidos por los niños en edad escolar de la comunidad de Madrid corresponden a aquellos con un alto contenido de ácidos grasos saturados, los cuales superaban las recomendaciones del Ministerio de Salud. Otros autores como Marín et al. (21) en 2014 encontraron asociación entre el incremento del peso en los niños de México y la escasa variedad y menor consumo de alimentos de menor aporte energético, como lo son las frutas y verduras. Esto concuerda con los resultados del estudio de la ciudad de Taltal en materia de refrigerios y alimentos extras, puesto que solo el $7,5 \%$ de los escolares estudiados consumía alimentos saludables como frutas y verduras; el resto tenía preferencia por el 
consumo de alimentos enlatados y procesados, así como de bebidas industrializadas con altos contenidos de azúcares refinados.

Algunos autores han encontrado altos porcentajes de consumo de los refrigerios y alimentos extras en los estudiantes y han reportado hasta un $26 \%$ de alimentos con alto contenido de grasas saturadas que incluían papas fritas, chocolates, entre otros. De ahí la importancia de la educación a las madres, por ser ellas las encargadas más frecuentes de proveer desde las casas este tipo de alimentos al menor. Situaciones similares encontraron Contreras et al. (22) al evaluar los alimentos incluidos en la lonchera de niños estudiantes colombianos del nivel de transición, lo que evidenció un elevado contenido de energía, proteína, grasa y carbohidratos, alcanzando un aporte diario de $416 \mathrm{kcal}$.

En Chile, estudios como el de Araneda et al. (23) reportaron las preferencias de los niños, quienes al momento de elegir sus refrigerios privilegian su interés en un alto consumo de líquidos de alta densidad energética como lo son las bebidas azucaradas, y el bajo consumo de agua. Dichos autores relacionaron la prioridad de los niños por los alimentos poco saludables aludiendo que por ser más placenteros y apreciados por los pares les ayuda a integrarse al grupo social. Situación similar se reporta en el presente estudio, porque, independiente del sexo, más del $30 \%$ de los escolares prefirieron refrigerios altos en grasa, incluidas las bebidas gaseosas azucaradas.

En materia de actividades físicas, en el presente estudio no hay diferencias estadísticamente significativas entre hombres y mujeres, aunque los hombres dedican más tiempo a los juegos recreativos diarios y a las actividades físicas sistemáticas semanales. No obstante, la tendencia de la actividad física por sexo es semejante a lo encontrado por Nava et al. (24), quienes sí reportaron diferencias estadísticamente significativas entre hombres y mujeres en edad escolar.

En relación con las actividades diarias como caminar, el presente estudio evidencia que - del total de la muestra- el 53,2\% camina menos de dos cuadras al día, resultados similares a los encontrados por otros autores como Atalah et al. (25), quienes reportaron, en niños de Aysén, Chile, que el número de cuadras caminadas por ellos era bajo: el $16 \%$ prácticamente no caminaba, la mitad de los niños participaba regularmente en juegos activos al aire libre y no más de un tercio practicaba deportes.

El 64,8\% de los escolares estudiados dedica de dos a tres horas a la actividad física sistemática, situación algo diferente a lo reportado en el año 2008 por Burrows et al. (10), quienes, al explorar los hábitos de ingesta y actividad física en escolares, encontraron un $68,6 \%$ de las niñas y un $59,9 \%$ de los niños con menos de dos horas dedicadas a la actividad física sistemática.

En lo relacionado con las conductas de menor gasto de energía o conductas sedentarias, se observó que el 96,6\% del grupo estudiado tiene -independientemente del sexo- casi idéntica conducta sedentaria, caracterizada por las horas dedicadas a ver televisión y al uso de su celular por más de seis horas. Estas conductas son similares a aquellas descritas por Miranda (26) en 2017, quien encontró un 95,6\% - de un total de 165 menores-con dedicación suficiente de tiempo diario a actividades de menor gasto de energía como ver televisión y utilizar su computador, exponiéndose a un factor condicionante predictor de sobrepeso.

Esto también fue observado y reportado en el año 2015 por otros autores como Camargo et al. (27), quienes después de estudiar la confiabilidad de un cuestionario para medir la actividad física y los comportamientos sedentarios en niños desde 
preescolar hasta cuarto grado de primaria hallaron comportamientos sedentarios que alcanzaron un $99,4 \%$ para el uso del televisor y un $74,6 \%$ para el uso del computador.

La anterior tendencia también fue evidenciada por González et al. (28), quienes, al estudiar los factores predisponentes, reforzadores y capacitadores para una alimentación y actividad física adecuadas en escolares de Sonora, México, encontraron en el $100 \%$ de los encuestados preferencia por el uso de tecnología por sobre la actividad física, por lo que esta tendencia se asocia con el estilo de la crianza recibida de sus padres, debido, en parte, a la restricción o carencia de alimentos industrializados y uso de videojuegos, entre otros factores.

Ellos identificaron como factor predisponente para una alimentación y actividad física inadecuadas el acceso ilimitado a los alimentos industrializados y a los videojuegos. Los autores consideran que el conocimiento generado respecto a los escolares permitirá planificar con fundamento aquellas medidas de promoción y prevención necesarias para abordar el problema estudiado, evitar complicaciones en salud a futuro y aportar al control del problema en salud pública inherente a las enfermedades crónicas no trasmisibles secundarias a un estado nutricional inadecuado.

En conclusión, respecto a la evaluación antropométrica, la mitad de los estudiantes no calificaron como normales (según IMC), la mayoría de ellos presentó sobrepeso y obesidad siendo más afectado el grupo de hombres.
Según la evaluación del consumo de alimentos, en ambos sexos todas las combinaciones estudiadas presentan preferencia por alimentos altos en grasa.

Acorde a los hallazgos de actividad física, no existe diferencia entre sexos: el $50 \%$ de ellos camina menos de cinco cuadras diarias y dedican la mayor parte del tiempo a actividades de gasto calórico mínimo y la actividad física sistemática que practican es solo la realizada en el colegio.

En el presente estudio solo se observa una asociación simple (no determinante de causalidad) entre la alta prevalencia de obesidad-sobrepeso de los escolares de primer año básico de la ciudad de Taltal: la calidad y cantidad de alimentos ingeridos y la escasa práctica de actividad física en el colegio y fuera de él.

Por lo señalado, los autores del presente artículo plantearon al alcalde y a los concejales de la ciudad una propuesta inicial de medidas de intervención basada en los hallazgos de la población escolar estudiada y en la evidencia científica disponible, orientada a la capacitación, coaching motivacional de padres y fundamentos para la generación de programas de educación para la salud (29).

\section{CONFLICTO DE INTERESES}

Los autores del presente artículo declaran no tener conflictos de interés de ninguna naturaleza, que hayan afectado la recopilación de información, presentación de resultados y conclusiones del tema abordado en el presente artículo.

\section{-Referencias}

1. ACC/SCN. Stunting and young child development. En: 3rd Report on the World Nutrition Situation. Switzerland: World Health Organization; 1997, pp. 7-24.

2. Yáñez R, Olivares S, Torres I, Guevara M, Díaz N. Consumo de alimentos en escolares chilenos. Su relación con las guías y la pirámide alimentaria. Rev Chil Nutr. 2001;28:422-8. 
3. Chile, Junta Nacional de Auxilio Escolar y Becas. Informe mapa nutricional 2015. [Internet]. [Citado noviembre de 2018]. Disponible en: https://www.junaeb.cl/wp-content/uploads/2017/07/Informe-Mapa-Nutricional-2015.pdf

4. Olivares S, Kain J, Lera L, Pizarro F, Vio F, Morón C. Nutritional status, food consumption and physical activity among Chilean school children. A descriptive study. Eu J Clin Nutr. 2004;58(9):1278-85. DOI: 10.1038/sj.ejcn.16019625

5. Vio F, Salinas J. Promoción de la salud y calidad de vida en Chile: una política con nuevos desafíos. Rev. Chilena de Nutrición. 2006;33(supl.1):252-9. DOI: 10.4067/S0717-75182006000300006

6. Méndez M, Estay J, Calzadilla A, Durán S, Díaz P. Comparación del desarrollo psicomotor en preescolares chilenos con normopeso versus sobrepeso/obesidad. Nutr Hosp. 2015;32(1):151-5. DOI: 10.3305/nh.2015.32.1.9060

7. República de Chile, Ministerio del Deporte de Chile. Encuesta Nacional de Hábitos de Actividad Física y Deportes en la población de 18 años y más. [Internet]. [Citado enero de 2019]. Disponible en: http://www.mindep.cl/encuesta-actividadfisica-y-deporte-2018/

8. República de Chile, Ministerio de Salud Pública. División de Políticas Públicas Saludables y Promoción. Departamento de Nutrición y Alimentos. MINSAL 2016. [Internet]. [Citado agosto de 2018]. Disponible en: http://www.bibliotecaminsal.cl/wp/ wp-content/uploads/2018/03/2018.03.16-Patrones-de-crecimiento-para-la-evaluaci\%C3\%B3n-nutricional-de-ni\%C3\%B10sni\%C3\%B1as-y-adolescentes-2018.pdf

9. Olivares S, Araneda J, Morales G, Leyton B, Bustos N, Hernández M, et al. Actitudes de escolares chilenos de distinto nivel socioeconómico al inicio de la implementación de la ley que regula la venta y publicidad de alimentos altos en nutrientes críticos. Nutr. Hosp. 2017;34(2):431-8. DOl: 10.20960/nh.499

10. Burrows R, Díaz E, Sciaraffia V, Gattas V, Montoya A, Lera L. Hábitos de ingesta y actividad física en escolares, según tipo de establecimiento al que asisten. Rev Méd Chile. 2008;136(1):53-63. DOI: 10.4067/S0034-98872008000100007

11. Ricciardi R. Sedentarism: a concept analysis. Nursing Forum. 2005;40:79-87.

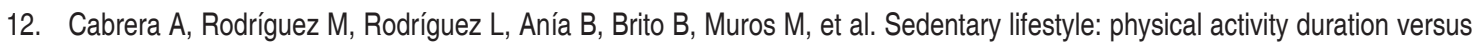
percentage of energy expenditure. Rev Esp Cardiol. 2007;60(3):231-3.

13. Alarcón MH, Lancellotti DA, Pedreros AR, Bugueño CA, Munizaga RA. Estado nutricional y composición corporal en escolares de La Serena, Chile. Rev Chil Nutr. 2016;43:138-45. DOI: 10.4067/S0717-75182016000200005

14. República de Chile, Junta Nacional de Auxilio Escolar y Becas. Informe mapa nutricional 2016. [Internet]. [Citado noviembre de 2018]. Disponible en: https://www.junaeb.cl/wp-content/uploads/2013/03/Informe-Mapa-Nutricional-2016.pdf

15. Ulloa N, Sapunar J, Bustos P, Sáez k, Asenjo S, Taibo M, et al. Epidemiología del sobrepeso y obesidad infanto-juvenil en las comunas de Concepción, Coronel y Hualpén, VIII Región de Chile. Rev Med Chil. 2010;138:1365-72. DOI: 10.4067/ S0034-98872010001200004

16. Benjumea MV, Molina DI, Arbeláez PE, Agudelo LM. Circunferencia de la cintura en niños y escolares manizaleños de 1 a 16 años. Rev Col Cardiol. 2008;15(1):23-34. Disponible en: http://www.scielo.org.co/pdf/rcca/v15n1/v15n1a5.pdf

17. Burrows R, Weisstaub G, Ceballos Z, Gaitas V, Leiva L, Lera L, et al. Síndrome metabólico en niños y adolescentes: asociación con sensibilidad insulínica, grado y distribución del sobrepeso. Rev Med Chile. 2007;135(2):174-81. DOI: 10.4067/ S0034-98872007000200005

18. Cruz ML, Weigensberg MJ, Huang TTK, Ball G, Shaibi GQ, Goran MI. The metabolic syndrome in overweight hispanic youth and the role of insulin sensitivity. J Clin Endocrinol Metab. 2004;89:108-13. DOI: 10.1210/jc.2003-031188

19. Lee S, Bacha F, Gungor N, Arslanian SA. Waist circumference is an independent predictor of insulin resistance in black and white youths. J Pediatr. 2006;148:188-94. DOI: 10.1016/j.jpeds.2005.10.001 


\section{Actividad física y alimentación en escolares chilenos}

20. Zarceño $\mathrm{C}$. Estudio de diferentes patrones de consumo de alimentos habituales y ocasionales al perfil lipídico de la dieta de los niños en edad escolar de la comunidad de Madrid. Percepción y conocimientos de la población [tesis doctoral]. Madrid: Universidad Complutense de Madrid; 2017. [Citado noviembre de 2108]. Disponible en: https://dialnet.unirioja.es/servlet/ tesis?codigo $=149101$

21. Marín AD, Sánchez G, Maza LL. Prevalencia de obesidad y hábitos alimentarios desde el enfoque de género: el caso de Dzutóh, Yucatán, México. Estud Soc. 2014;22(44):64-90. Disponible en: http://www.scielo.org.mx/scielo.php?script=sci_artt ext\&pid=S0188-45572014000200003

22. Fuentes EP. Relación entre el aporte energético de las loncheras y el índice de masa corporal en un grupo de niños de transición del Colegio Champagnat en la ciudad de Bogotá DC. [tesis de licenciatura]. Bogotá: Pontificia Universidad Javeriana, Facultad de Ciencias; 2014. [Consultado noviembre de 2018]. Disponible en: http://hdl.handle.net/10554/16059

23. Araneda J, Lobos L, Olivares S, Oliva P, Quezada G, Sandoval P. Bebidas azucaradas: representaciones de escolares con sobrepeso y obesidad. Rev Chil Nutr. 2017;44:276-282. DOI: 10.4067/s0717-75182017000300276

24. Nava MC, Pérez A, Herrera HA, Hernández R A. Hábitos alimentarios, actividad física y su relación con el estado nutricionalantropométrico de preescolares. Rev Chil Nutr. 2011;38(3):301-12. DOI:10.4067/S0717-75182011000300006

25. Atalah E, Urteaga C, Rebolledo A, Delfín S, Ramos R. Patrones alimentarios y de actividad física en escolares de la Región de Aysén. Rev Chil Pediatr. 1999;70(6):483-90. DOI: 10.4067/S0370-41061999000600005

26. Miranda-Rios L, Vásquez-Garibay EM, Romero-Velarde E, Nuño-Cosio ME, Campos-Barrera L, Caro-Sabido EA, et al. Factores asociados a la actividad física y el índice de masa corporal en escolares de Arandas, Jalisco, México. Rev Med Inst Mex Seguro Soc. 2017;55(4):472-80. Disponible en: https://www.medigraphic.com/pdfs/imss/im-2017/im174k.pdf

27. Camargo DM, Santisteban S, Paredes E, Flórez MA, Bueno D. Confiabilidad de un cuestionario para medir la actividad física y los comportamientos sedentarios en niños desde preescolar hasta cuarto grado de primaria. Biomédica. 2015;35(3):347-56. DOI: 10.7705/biomedica.v35i3.2502

28. González DG, Grijalva MI, Montiel M, Ortega MI. Identificación de factores predisponentes, reforzadores y capacitadores para una alimentación y actividad física adecuadas en escolares sonorenses. Región y Sociedad. 2018;30(72):1-33. DOI: 10.22198/rys.2018.72.a893

29. Aguilar MJ, Ortegón A, Mur N, Sánchez JC, García JJ, García I, et al. Programas de actividad física para reducir sobrepeso y obesidad en niños y adolescentes: revisión sistemática. Nutr Hosp. 2014;30(4):727-40. DOI: 10.3305/nh.2014.30.4.7680 
Anexo 1. Hábitos de ingesta

\begin{tabular}{|c|c|c|}
\hline Aspecto evaluado & Código & Opciones de respuesta \\
\hline 1.- ¿Cuántas comidas hace al día? & & 2 comidas \\
\hline Desayuno - Almuerzo - Once - Cena- & & 3 comidas \\
\hline Refrigerio & & 4 - 5 comidas \\
\hline \multirow{3}{*}{ 2.- Combinaciones desayuno/onces } & 0 & $\begin{array}{l}\text { Leche entera, pan amasado, hallulla, pan con queso, mantequilla o mortadela, té o } \\
\text { café puro, hotdog, pizza, productos de pastelería, huevo frito, manjar, paté, arrollado. }\end{array}$ \\
\hline & 1 & $\begin{array}{l}\text { Leche semidescremada, marraqueta, galletas agua/soda, mermelada, palta, } \\
\text { margarina, huevo duro, jamón de pavo, queso blanco, cereal azucarado. }\end{array}$ \\
\hline & 2 & $\begin{array}{l}\text { Leche o yogurt descremado, fruta, jugo de fruta natural, pan integral, cereales } \\
\text { naturales o integrales, quesillo, tomate. }\end{array}$ \\
\hline \multirow{3}{*}{ 3.-Combinaciones almuerzo/comida } & 0 & $\begin{array}{l}\text { Pastas (ravioles, lasaña, otros), tortillas, frituras, postres elaborados (flanes, gozzo } \\
\text { etc.), fruta en conserva, fruta con crema o miel, hotdog, hamburguesa, pizza, } \\
\text { pantrucas, bebida gaseosa, huevo frito o revuelto. }\end{array}$ \\
\hline & 1 & $\begin{array}{l}\text { Carnes, arroz, fideos, puré, pan, papas, sopas, compotas de frutas, jaleas, yo- } \\
\text { gurt, jugo en polvo, bebida diet. }\end{array}$ \\
\hline & 2 & $\begin{array}{l}\text { Ensaladas con verduras de todo tipo, guisos de verdura con carnes, pescado o } \\
\text { ave, cazuela, carbonada, leguminosas, postres de frutas, jugos naturales, agua. }\end{array}$ \\
\hline \multirow{3}{*}{ 4.- Refrigerio } & 0 & $\begin{array}{l}\text { Papas fritas u otras, pasteles, chocolates o confites, snack, helados, alfajores, } \\
\text { negritas, bebidas gaseosas, sopaipillas". }\end{array}$ \\
\hline & 1 & $\begin{array}{l}\text { Sándwich palta o pollo, galletas, nueces, galletones, cereales, jugos azucarados, } \\
\text { bebida light. }\end{array}$ \\
\hline & 2 & Fruta, yogurt, huevo duro. \\
\hline \multirow{3}{*}{ 5.- Alimentos extras } & 0 & $\begin{array}{l}\text { Dulces, chocolates, papas fritas y similares, bebidas gaseosas, productos de pas- } \\
\text { telería, snack, helados, sopaipillas, picarones"“; pasteles, sándwich jamón o queso. }\end{array}$ \\
\hline & 1 & Galletas, sándwich de avena, atún, mermelada, pan solo. \\
\hline & 2 & Fruta, jugos fruta, yogurt, agua. \\
\hline
\end{tabular}

Fuente: Adaptado de Burrows R, Díaz E, Sciaraffia V, Gattas V, Montoya A, Lera L. Hábitos de ingesta y actividad física en escolares, según tipo de establecimiento al que asisten. Rev Méd Chile. 2008;136(1):53-63. DOI: 10.4067/S0034-98872008000100007

0 es alto en grasa; 1 es moderado en grasa y 2 es bajo en grasa

* Gozzo es un postre elaborado a base de yogurth con chocolate y crema.

** Sopaipilla son masas fritas.

***Picarones son roscas de masa de harina de trigo. 
Actividad física y alimentación en escolares chilenos

Anexo 2. Encuesta de hábitos de actividad física

\begin{tabular}{l}
\hline Tipo de actividad: horario \\
\hline 1.- Acostado \\
\\
\\
2.- Actividades de gasto mínimo, sentado en las siguientes actividades: \\
$\begin{array}{ll}\text { a) Clases } & \text { a) } \\
\text { b) TV } & \text { b) } \\
\text { c) Tareas o estudio } & \text { c) } \\
\text { d) Computador o Nintendo } & \text { d) }\end{array}$
\end{tabular}

3.- Caminar: cuadras caminadas para trasladarse al colegio 0 a cualquier otro lugar al que van rutinariamente

Horas de actividad

Total $\mathrm{h}$ diarias

Total h diarias
a)
b)
c)
d)

Total cuadras diarias

4.- Juegos recreativos después de la jornada escolar (se excluyen los recreos) bicicleta, patines, fútbol, otros

Total $\mathrm{h}$ diarias

5.- Actividades sistemáticas

Total $\mathrm{h}$ semanales
a) Educación física
a)
b) Gimnasia aeróbica
b)
c) Fútbol
c)
d) Tenis
d)
e) Básquetbol
e)
f) Otros
f)

Fuente: adaptado de Burrows R, Díaz E, Sciaraffia V, Gattas V, Montoya A, Lera L. Hábitos de ingesta y actividad física en escolares, según tipo de establecimiento al que asisten. Rev Méd Chile. 2008;136(1):53-63. DOI: 10.4067/S0034-98872008000100007 